Short Communication

\title{
Metabolite profiling identifies the mycotoxin alternariol in the pathogen Stagonospora nodorum
}

Kar-Chun Tan ${ }^{1}$, Robert D. Trengove ${ }^{2,3}$, Garth L. Maker ${ }^{2,3}$, Richard P. Oliver ${ }^{1}$ and Peter S. Solomon ${ }^{1,3^{*}}$

Affiliations

${ }^{1}$ Australian Centre for Necrotrophic Fungal Pathogens, SABC, Division of Health Sciences, Murdoch University, Murdoch 6150, Australia.

${ }^{2}$ School of Pharmacy, Faculty of Health Sciences, Murdoch University, Murdoch 6150, Australia.

${ }^{3}$ Metabolomics Australia, Murdoch University, Perth 6150, WA, Australia.

*Corresponding author; p.solomon@murdoch.edu.au 
Abstract A recent comparative proteomics study identified that a short-chain dehydrogenase (Sch1) was required for asexual sporulation in the wheat fungal pathogen Stagonospora nodorum [Tan, K.-C., Heazlewood, J.L., Millar, A.H., Thomson, G., Oliver, R.P., and Solomon, P.S. (2008). A signalling-regulated short-chain dehydrogenase of Stagonospora nodorum regulates asexual development. Eukaryotic Cell, (in press)]. Metabolite profiling was undertaken on these mutant strains to help elucidate the role of Sch1. Gas chromatography-mass spectrometry of the polar metabolites in the Sch1 mutants identified the strong accumulation of a secondary metabolite that was at the level of detection in the wild-type strains. Comparative analysis of the secondary metabolite and the postharvest mycotoxin alternariol confirmed the identity of the compound. This is the first report to confirm the presence of a mycotoxin in S. nodorum. 


\section{Introduction}

Stagonospora (syn. Septoria) nodorum (Berk.) Castell. and Germano [teleomorph: Phaeosphaeria (syn. Leptosphaeria) nodorum (Müll.) Hedjar.] is a major pathogen of wheat and other cereals (Solomon et al., 2006d) causing stagonospora nodorum blotch (previously septoria nodorum blotch and glume blotch). Previous studies focused on understanding the disease have demonstrated critical roles for host-specific toxins and various primary metabolic pathways for effective fungal pathogenicity (Solomon et al., 2004a; Solomon et al., 2005a; Solomon et al., 2006a; Solomon et al., 2006e).

Signal transduction pathways within $S$. nodorum also have essential roles in pathogenicity (Solomon et al., 2004b; Solomon et al., 2005b; Solomon et al., 2006b). Each of the cAMPdependent, the MAP kinase and the $\mathrm{Ca} 2+$ signalling pathways all have demonstrated roles in enabling $S$. nodorum to complete its pathogenic lifecyle. The downstream genes and proteins regulated by these signalling pathways are poorly defined. Recent studies in S. nodorum have used comparative proteomics to identify Sch1, a gene encoding a hypothetical short-chain dehydrogenase that is positively regulated by the Ga subunit, Gna1 (Tan et al., 2008). Subsequent characterisation of the strains of S. nodorum lacking Sch1 showed that the gene was required for asexual development. Detailed transcript and histological analysis showed that Sch1 was required for the maturation of the asexual fruiting body pycnidia, although the precise link was not discovered.

In this study, we exploit the power of metabolomics to compare the metabolome of the Sch1 mutants with the wild-type to investigate the function of this gene. Metabolomics has many well documented strengths that complement the existing 'omics technologies with metabolic information that may be viewed as better reflecting biological endpoints (Harrigan et al., 2007). Additionally, metabolomics is well suited as a high-throughput tool to screen 
genetic variation compared to the other 'omics technologies. Sample preparation is comparatively simple, the cost is generally affordable and it does not require access to a deeply annotated genome sequence making it an ideal tool for non-model organisms. Thus the aim of this study was to use metabolomics to shed light on the role of Sch1 in the pathogen S. nodorum. 


\section{Experimental methods}

2.1 Growth and maintenance of fungal strains

S. nodorum wild-type SN15 was obtained from Department of Agriculture, Western Australia. The sch1 mutants used during this study were developed and described previously (Tan et al., 2008). All fungal cultures were maintained on artificial agar media as described elsewhere (Solomon et al., 2004c).

2.2 GC-MS metabolite analysis

Metabolite analysis of S. nodorum was adapted from previous studies (Solomon et al., 2006c;

Solomon et al., 2006e; Lowe et al., 2008). Fungal mycelia were scrapped from plates using a sterile scapel blade (No. 10) into a pre-weighed $2 \mathrm{~mL}$ microfuge tube. The tube was immediately immersed in liquid nitrogen prior to freeze drying and being re-weighed. Approximately $10 \mathrm{mg}$ of dry sample was then used for metabolite extraction. $700 \mu \mathrm{l}$ of $-40 \mathrm{oC}$ pure methanol was added to the mycelia and vortexed briefly. The tube was then placed in liquid nitrogen and then thawed on ice. The mycelia were then separated from the extracted metabolites by centrifugation at $21,000 \mathrm{~g}$ for $2 \mathrm{~min}$ and $4 \mathrm{oC}$. The supernatant was removed and placed in a new $2 \mathrm{~mL}$ microfuge tube whilst a further $700 \mu \mathrm{lof}-40 \mathrm{oC}$ pure methanol was added to the mycelia and the freeze/thaw process repeated. The mycelia suspension was again centrifuged and the supernatant was added to the original supernatant. $50 \mu \mathrm{L}$ of a ribitol standard $(1.3 \mathrm{mM})$ was added and the extracted metabolites were freeze dried prior to derivatisation. 
Methoximation of carbonyl groups was performed by addition of $50 \mu \mathrm{L}$ methoxylamine$\mathrm{HCl}\left(20 \mathrm{mg} / \mathrm{mL}\right.$ in pyridine) to the dried metabolites followed by incubation at $30^{\circ} \mathrm{C}$ for 90 minutes with shaking. Trimethylsilyl (TMS) esters were then created by addition of $80 \mu \mathrm{L} \mathrm{N}$ trimethylsilyl- $N$-methyl trifluoroacetamide (MSTFA) and incubation at $37^{\circ} \mathrm{C}$ for 30 minutes with shaking.

For the GC-MS analysis, samples were injected as $1 \mu \mathrm{L}$ derivatised metabolites in a 20:1 split ratio. The GC-MS equipment consisted of an Agilent 7680 autosampler, an Agilent 6890 gas chromatograph and an Agilent 5973N quadrupole mass spectrometer (Agilent, Palo Alto, CA, USA). The GC-MS system was auto-tuned using perflurotributylamine (PFTBA). A 30m Varian VF-5ms column with a 10m integrated Varian EZ-Guard column was use for the gas chromatography (Varian, Palo Alto, CA, USA). Injection temperature was $230^{\circ} \mathrm{C}$, interface temperature was $300^{\circ} \mathrm{C}$ and the ion source temperature was $230^{\circ} \mathrm{C}$. The carrier gas (helium) flow rate was retention-time locked to elute mannitol-TMS at 30.6 minutes. The temperature gradient consisted of an initial temperature of $70^{\circ} \mathrm{C}$ increasing $1{ }^{\circ} \mathrm{C}$ per minute for 5 minutes before increasing to a final temperature of $300^{\circ} \mathrm{C}$ at a temperature ramp rate of $5.6^{\circ} \mathrm{C}$ per minute. Mass spectra and chromatograms were normalized to the ribitol internal standard and the weight of the sample and processed using AnalyzerPro (SpectralWorks Ltd, Runcorn, U.K.) employing the MatrixAnalyser function. For each strain, between six and eight biological replicates were used.

Data analyses were undertaken using The Unscrambler (Camo) and the JMP7 package (SAS Institute). Statistical significance was determined using the Tukey-Kramer analysis. Principal Component Analysis of the GC-MS data was performed subsequent to $\log (10)$ transformation of the normalised abundance data.

\subsection{ESI-MS/MS}


Samples were prepared as above with the exception that ribitol was not included. The dried extracts were redissolved in 50:50 acetonitrile:water with $0.1 \%$ formic acid and infused at 5 $\mu \mathrm{l} /$ min into a Varian 320-MS triple quadrupole mass spectrometer. Nebulising gas $\left(\mathrm{N}_{2}\right)$ pressure was $15 \mathrm{psi}$, while drying gas $\left(\mathrm{N}_{2}\right)$ was at $10 \mathrm{psi}$ and $300^{\circ} \mathrm{C}$. Electrospray needle voltage was $5000 \mathrm{~V}$, shield voltage was $600 \mathrm{~V}$ and capillary voltage was $65 \mathrm{~V}$. Scan range was 100 to $300 \mathrm{amu}$, with a scan time of 0.5 seconds. Automated MS/MS breakdown analysis was performed (using argon as collision gas) with the optimal collision energy determined to be -11 V. Data was analysed using Varian MS Workstation 6.9. 


\section{Results and discussion}

A non-targeted metabolomics approach was used to characterise the Sch1 mutation in $S$. nodorum. Samples were prepared for GC-MS analysis as described above. S. nodorum SN15, Sch1-30, sch1-11 and sch1-42 metabolite extracts were each analysed in triplicate. Data collected from the GC-MS was processed using the AnalyzerPro (SpectralWorks, U.K.) software package. Representative chromatograms displaying total ion counts (TIC) are shown in Fig. 1. Approximately 223 metabolites, both of known and unknown identity, were resolved under the separation conditions. Immediately apparent was the accumulation of a late eluting unknown compound at 45.57min (named RT4557) in the sch1 strains, which was at or close to the level of detection in the wild type traces.

The abundances of each metabolite in all strains were subjected to multivariate analysis. The Principal Component Analysis (PCA) score plot for PC1 and PC2 is shown in Fig. 2a. PC1 captured $88 \%$ variance and clustered the wild-type and ectopic strains from the mutants. PC2 accounted for $6 \%$ of the variance. Factor loadings of PC1 revealed it to be predominantly influenced by RT4557 (data not shown). Glutamate, glutamine and pyroglutamate also contributed to $\mathrm{PCl}$ although statistical analysis of the compounds influencing PC1 found that only RT4557 differed significantly when comparing the four strains. A comparison of the relative abundance of RT4557 found it to accumulate in the sch1 mutants to approximately 200-fold the levels measured in SN15 and Sch1-30 (Fig. 2b).

A comparison of the RT4557 fragmentation pattern with existing libraries (NIST05 and Wiley) was unable to determine the identity of the compound. Consequently, a series of complementary analyses were undertaken to resolve its identity. Firstly, the mass of the derivatised parental ion was sought using chemical ionisation (CI). Positive CI using methane yielded a base peak of $475 \mathrm{~m} / \mathrm{z}$ compared to $459 / \mathrm{z}$ base peak for electron ionisation (data not 
shown). The mass of the derivatised RT4557 was determined as 474 as this species is singularly charged. Allowing for the additional mass due to the presence of trimethyl silyl group(s), the molecular weight of the underivatised RT4557 was likely to be either 402 (1TMS), 330 (2TMS), 258 (3TMS) or 186 (4TMS).

RT4557 was then purified using liquid chromatography with a $\mathrm{C}_{18}$ column. A large peak eluting at a retention time of 27 mins was present in the sch1 extracts but not SN15 (data not shown). These fractions were collected with an aliquot derivatised and re-analysed using GCMS to confirm the identity of the peak as RT4557. A large peak was identified at retention time of $45.57 \mathrm{~min}$. The fragmentation pattern of the compound was almost identical to the previously identified RT4557, albeit appearing less fragmented. Subsequent analysis of this cleaner fragmentation pattern using AnalyzerPro and the NIST05 database revealed a significant match to 3,7,9-Tris[(trimethylsilyl)oxy]-1-methyl $(6 \mathrm{H})$ dibenzo[b,d]pyran-6-one, also known as the mycotoxin alternariol.

To confirm the identity of RT4557 as alternariol, a small amount of standard was purchased and subjected to direct infusion electrospray ionisation-mass spectrometry (ESIMS) using a Varian 320-MS triple quadrupole mass spectrometer (Fig.3). A peak corresponding to $259 \mathrm{~m} / \mathrm{z}$ was present. Allowing for the compound to be singularly charged, this corresponds to a mass of $258 \mathrm{Da}$, the empirical mass for alternariol. A more abundant ion corresponding to an acetonitrile adduct was also apparent at $288 \mathrm{~m} / \mathrm{z}$. Direct infusion of RT4557 compound after liquid chromatography revealed an identical pattern with peaks at $259 \mathrm{~m} / \mathrm{z}$ and $288 \mathrm{~m} / \mathrm{z}$ identified providing further evidence of the identity of RT4557 as alternariol. A mass of $258 \mathrm{Da}$ correlates well with the chemical ionisation data. Fragmentation of the $259 \mathrm{~m} / \mathrm{z}$ ion identified from the RT4557 sample revealed a near identical fragmentation pattern to the standard further substantiating the identity of RT4557 
as alternariol as well as previously published MS/MS data for the compound (Scott et al., 1997).

Alternariol is a post-harvest toxin commonly associated with the genus Alternaria and is known to be found in various fruits, cereals and processed fruit products (Scott, 2001). The consumption of foods contaminated with alternaria toxins including alternariol has been previously associated with esophageal cancer with other reports commenting on its mutagenicity and toxicity (Brugger et al., 2006; Lehmann et al., 2006; Pfeiffer et al., 2007). The toxins altenuene and tenuazonic acid, often associated with alternaria infections, were not detected during the course of this study (data not shown). To our knowledge alternariol has never been detected on wheat or other grains, but this study highlights to need to broaden the screening of agricultural products for natural toxins.

This study has provided 2 significant novel findings. Firstly, mycotoxins have until now yet to be identified in S. nodorum. S. nodorum is associated with significant yield losses throughout the world. Identifying this pathogen as a mycotoxin producer is novel and will require experts involved in managing the disease to now consider this. No longer can $S$. nodorum be singularly associated with inflicting yield losses and it should now be considered a health risk and treated as such.

The second novel finding from this study is the involvement of a short-chain dehydrogenase in the synthesis of alternariol. Surprisingly, the synthesis of alternariol in vivo is poorly understood with none of the genes involved yet to be identified. Sch1 is a short chain dehydrogenase of unknown function but has a demonstrated role in maturation of asexual sporulation structures known as pycnidia (Tan et al., 2008). At this point, it is difficult to comment on putative roles of Sch1 apart from speculating on its role as a potential negative regulator of alternariol production. However, further studies are required to confirm this. Worth mentioning that $\mathrm{Ga}$ mutant accumulate DOPA - together suggests that 
coordinated cellulare development and gene expression is needed for the regulated formation of viable pycinidia. 


\section{Conclusion}

In this study, a series of complementary platforms have been to demonstrate for the first time that $S$. nodorum has the capacity to synthesize mycotoxins. Such a finding highlights the power of a non-biased metabolomics approach and has shed new light on this disease. Alternariol is a post-harvest mycotoxin and studies are now underway correlating the presence of alternariol with $S$. nodorum infections in post-harvest fields and also stored grain. 
Acknowledgements This research was supported by the Grains Research and Development Corporation. K.-C.T. was funded by an Australian Postgraduate Award. 
References

Brugger, E.M., Wagner, J., Schumacher, D.M., Koch, K., Podlech, J., Metzler, M., and Lehmann, L. (2006). Mutagenicity of the mycotoxin alternariol in cultured mammalian cells. Toxicology Letters 164, 221-230.

Harrigan, G.G., Martino-Catt, S., and Glenn, K.C. (2007). Metabolomics, metabolic diversity and genetic variation in crops. Metabolomics 3, 259-272.

Lehmann, L., Wagner, J., and Metzler, M. (2006). Estrogenic and clastogenic potential of the mycotoxin alternariol in cultured mammalian cells. Food and Chemical Toxicology 44, 398-408.

Lowe, R.G.T., Lord, M., Rybak, K., Trengove, R.D., Oliver, R.P., and Solomon, P.S. (2008). A metabolomic approach to dissecting osmotic stress in the wheat pathogen Stagonospora nodorum. Fungal Genetics and Biology, (in press).

Pfeiffer, E., Schebb, N.H., Podlech, J., and Metzler, M. (2007). Novel oxidative in vitro metabolites of the mycotoxins alternariol and alternariol methyl ether. Molecular Nutrition and Food Research 51, 307-316.

Scott, P.M. (2001). Analysis of agricultural commodities and foods for Alternaria mycotoxins. Journal of AOAC International 84, 1809-1817.

Scott, P.M., Weber, D., and Kanhere, S.R. (1997). Gas chromatography-mass spectrometry of Alternaria mycotoxin. Journal of Chromatography A 765, 255-263.

Solomon, P.S., Tan, K.-C., and Oliver, R.P. (2005a). Mannitol 1-phosphate metabolism is required for sporulation in planta of the wheat pathogen Stagonospora nodorum. Molecular Plant-Microbe Interactions 18, 110-115.

Solomon, P.S., Joergens, C.I., and Oliver, R.P. (2006a). $\delta$-Aminolevulinic acid synthesis is required for virulence of the wheat pathogen Stagonospora nodorum. Microbiology 152, 1533-1538.

Solomon, P.S., Lee, R.C., Wilson, T.J.G., and Oliver, R.P. (2004a). Pathogenicity of Stagonospora nodorum requires malate synthase. Mol. Microbiol. 53, 1065-1073.

Solomon, P.S., Rybak, K., Trengove, R.D., and Oliver, R.P. (2006b). Investigating the role of calcium/calmodulin-dependent protein signalling in Stagonospora nodorum. Mol. Microbiol. 62, 367-381.

Solomon, P.S., Tan, K.-C., Sanchez, P., Cooper, R.M., and Oliver, R.P. (2004b). The disruption of a $\mathrm{G} \alpha$ subunit sheds new light on the pathogenicity of Stagonospora nodorum on wheat. Molecular Plant-Microbe Interactions 17, 456-466.

Solomon, P.S., Tan, K.-C., Sanchez, P., Cooper, R.M., and Oliver, R.P. (2004c). The disruption of a $\mathrm{G} \alpha$ subunit sheds new light on the pathogenicity of Stagonospora nodorum on wheat. Mol. Plant-Microbe Interact. 17, 456-466.

Solomon, P.S., Waters, O.D.C., Simmonds, J., Cooper, R.M., and Oliver, R.P. (2005b). The Mak2 MAP kinase signal transduction pathway is required for pathogenicity in Stagonospora nodorum. Current Genetics 48, 60-68.

Solomon, P.S., Lowe, R.G.T., Trengove, R.D., Rechberger, J., and Oliver, R.P. (2006c). Normalisation of metabolites in heterogenous systems using genomics. Analytical Biochemistry 350, 156-158.

Solomon, P.S., Lowe, R.G.T., Tan, K.-C., Waters, O.D.C., Bailey, A., and Oliver, R.P. (2006d). Stagonospora nodorum; cause of Septoria nodorum blotch of wheat. Mol. Plant Pathol. 7, 147-156.

Solomon, P.S., Waters, O.D.C., Joergens, C.I., Lowe, R.G.T., Rechberger, J., Trengove, R.D., and Oliver, R.P. (2006e). Mannitol is required for asexual sporulation in the 
wheat pathogen Stagonospora nodorum (glume blotch). Biochemical Journal 399, 231-239.

Tan, K.-C., Heazlewood, J.L., Millar, A.H., Thomson, G., Oliver, R.P., and Solomon, P.S. (2008). A signalling-regulated short-chain dehydrogenase of Stagonospora nodorum regulates asexual development. Eukaryotic Cell, (in press). 


\section{Figure legends}

Fig. 1 Total ion chromatograms (TIC) of polar extracts from SN15 (A), Sch1-30 (B), sch1-11 (C) and sch1-42 (D). The y-axes are log-scaled.

Fig. 2. (A) PCA scores plot of SN15 (匹), Sch1-30 (口), sch1-11 (•) and $\operatorname{sch} 1-42$ (०). (B) Histogram representing the relative abundances of RT4557 in each if the $S$. nodorum strains.

Fig. 3. ESI-MS chromatograms of partially purified RT4557 (A) and alternariol standard (B). The inserts represent MS/MS spectra collected on the $259 \mathrm{~m} / \mathrm{z}$ ion. 